\title{
Integrated Marker-Based Watershed Segmentation with Hybrid median Filter \\ Amanpreet kaur ${ }^{1}$, Ashish Verma ${ }^{2}$ \\ Ssiet,Derabassi (Pb.) India. \\ er.amanpreetk@gmail.com ${ }^{1}$ ashish.ver@gmail.com²
}

\begin{abstract}
This paper focuses on marker based watershed segmentation algorithms. As marker based watershed segmentation algorithm causes over segmentation and cause noise in the image produced. So to reduce these problems, different researchers has proposed different solutions, but the best solution so far found is to use bilateral filter. But bilateral filter fails to remove salt and pepper noise, so to overcome this problem hybrid median is integrated with marker based watershed algorithm. By comparing the proposed algorithm with existing algorithm we have seen a significant improvement in results of the proposed algorithm. It is found that the proposed algorithm becomes more useful when salt and pepper noise is present in the images.
\end{abstract}

\section{Indexing terms/Keywords}

External markers; Gradient; Internal markers; Image segmentation; Watershed.

\section{Academic Discipline And Sub-Disciplines}

Masters in Computer science engg.

\section{SUBJECT CLASSIFICATION}

Digital Image Processing

\section{Council for Innovative Research}

Peer Review Research Publishing System

Journal: INTERNATIONAL JOURNAL OF COMPUTERS \& TECHNOLOGY

Vol 11, No.2

editor@cirworld.com

www.cirworld.com, member.cirworld.com 


\section{LITERATURE SURVEY}

Boren Li, Mao Pan and Zixing Wu (2012) [1] has presented an approach to reduce over segmentation using both pre- and post-processing for watershed segmentation. They have used more prior knowledge in pre processing and merge the redundant minimal regions in post processing. In the initial stage of the watershed transform produces a gradient image from the original image, but also introduces the texture gradient. The texture gradient can be extracted using a gray -level co-occurrence matrix. Then, both gradient images are fused to give the final gradient image. After the initial results of segmentation, we use the merging region technique to remove small regions.Xiaoyan Zhang, Lichao Chen, Lihu Pan and Lizhi Xiong (2012) [2] has presented an image segmentation approach based on independent component analysis(ICA) and watershed algorithm is proposed. ICA is a method of image filtering for its characteristics.ICA can effectively remove the noises and maintain a clear image texture. Using independent component analysis algorithm can eliminate wavebands redundancy and extract the image signal components from source signal for image segmentation. Results showed that this method can process image segmentation effectively and identify features accurately.Zhonglin Xia ,Danfeng Hu and Xueyan Hu (2011) [3] has presented watershed algorithm is introduced based on image processing technology for the contamination of insulator. This algorithm can be used to obtain the size of contamination area of the insulator, and follow the following steps. Firstly segment the insulator image with watershed algorithm, and then the region growing algorithm is used to process the segmented image, which can guarantee the segmentation effectiveness and reduce the number of segmented regions so as to enhance the segmentation results of the insulator image. Li Cheng, Li Yan and Fan Shangchun (2011) [4] has presented a watershed algorithm as a primary tool of mathematical morphology for image segmentation is used. Based on the mathematical morphology, this paper develops a general purpose watershed segmentation algorithm used for the automatic segmentation in regions of pedestrians of infrared image. Then the experimental system is established by using the infrared CCD device for pedestrian image detection. The image segmentation results that the watershed method can implement the automatic detection and segmentation for the characteristic information in regions of interest of image.

Quan Longzhe and Jiang Enchen (2011) [5] has presented the improvement to the watershed algorithm and resolve the problems of 'over-segmentation' and 'leakage', and realized automatic segmentation of touching corn kernels. Firstly to get the pretreatment image, Wiener filter and mathematical morphology were adopted to reduce noises and clarify background of the image. Secondly, by combining with edge detection operators, the boundary of touching corn kernels was determined in the image, which can serve as watershed of the algorithm. Thirdly, erode transform was used to construct basin of the algorithm. At last, the algorithm of purifying image background was proposed, solving the problem of 'leakage'. Gui-Mei Zhang, Ming-Ming Zhou and Jun Chu (2011) [6] has presented image segmentation algorithm using reconstruction labeling watershed transform is presented. Because the proposed algorithm converts RGB color image into a new color image, and extracts specular quasi-invariant and shadow quasi-invariant to calculate the morphological gradient, the over-segmentation caused by specularities and shadows are solved. The morphological opening and closing with reconstruction is used to extract the markers and then applied watershed transform.

Jun Tang (2010) [7] has presented a color image segmentation method using seed region segmentation method with the combination of the watershed algorithm. Human eyes can distinguish thousands of colors but can only distinguish 20 kinds of grayscale, so we can easily and accurately find the target from the color images.It is difficult to find out from the grayscale image. The reason is that color can provide more information than grayscale. At present, specifically applied to the color image segmentation approach is not so much as for the gray-scale images, most of proposed color image segmentation methods are the combination of the existing grayscale image segmentation method on the basis of different color space. These methods are used to obtain better segmentation results. Chen Wei-bin1 and Zhang Xin2 (2010) [8] has focuses on segmentation of touching cell images which is helpful in recognising morphological structural model of touching cell. A morphological reconstruction is approach is used based on watershed transform. Morphological reconstruction is based on markers. Internal markers and external markers are used mark the regions of the image. Then watershed segmentation algorithm is applied and obtained satisfied results it was compared with traditional techniques and the new approach is better than the previous oneYuqian Zhao, Jianxin Liu, Huifen Li and Guiyuan Li (2008) [9] has presented a method for segmentation utilizing watershed algorithm based on morphological opening- closing filtering and distance transformation. The application of this method for segmenting dowels image overcomes over-segmentation effectively, and produces a satisfactory segmentation result apparently superior to the approach of employing watershed simply based on distance transformation

Shiping Zhu, Xi Xia, Qingrong Zhang and Kamel Belloulata (2008) [10] has presented an edge detection and image segmentation algorithm in image processing based on threshold segmentation is proposed. Aimed at that only one threshold or several thresholds are set in traditional threshold-based segmentation algorithm ,a new segmentation algorithm that each pixel in the image has its own threshold is proposed. In this algorithm, the threshold of a pixel in an image is estimated by calculating the mean of the grayscale values of its neighbor pixels, and the square variance of the grayscale values of the neighbour pixels are also calculated as an additional judge condition, so that the result of the proposed algorithm is the edge of the image. In fact the proposed algorithm is equal to an edge detector in image processing. Wei Zhang and Daling Jiang (2011) [11] has presented watershed transform a powerful morphological tool for image segmentation has been used. But it causes over segmentation and cause noise in the image produced. So they used marker based watershed technique to reduce noise and over segmentation. Based on marker watershed segmentation method, it first goes bilateral filtering for image processing, which is good to reduce the part of the small impact of noise on the post-processing and followed by use of distance transform and shape reconstruction method for image processing, making the next segmentation results with a more accurate positioning capability profile. Finally, the 
marker-based watershed transformation effectively reduces the over-segmentation problem and extracts a more precise outline of the area.

\section{INTRODUCTION}

The image segmentation is significance problem in different fields of computer vision and image processing. Image segmentation is the process of partitioning a digital image into multiple segments knows as set of pixels. The goal of segmentation is to simplify change the representation of an image into something that is more meaningful and easier to analyze. Image segmentation is typically used to locate objects and boundaries (lines, curves, etc.) in images. It is the process of assigning a label to every pixel in an image such that pixels with the same label share certain visual characteristics. The result of image segmentation is a set of segments that collectively cover the entire image, or a set of contours extracted from the image. Each of the pixels in a region are similar with respect to some characteristic or computed property such as color, intensity, or texture. The goal of segmentation operation is to simplify the image without discarding important image features. Image segmentation process in three stages. The first is image pre-processing, in this stage remove useless information from the image. The second stage is initial object discrimination, where objects are grossly separated into groups with similar attributes. Third stage is object boundary clean up, where object boundaries are reduced to single-pixel widths. In recent years several popular methods have been developed for image segmentation.

\section{A. THRESHOLDING METHOD}

The simplest method of image segmentation is called the thresholding method. This method is based on a threshold value to turn a gray-scale image into a binary image.

\section{B. SPLIT AND MERGE METHOD}

This method is used for the division of the image. If it is found non-uniform (not homogeneous), then it is split into four sonsquares (the splitting process), and so on so forth. Conversely, if four son-squares are homogeneous, they can be merged as several connected components (the merging process). This process continues recursively until no further splits or merges are possible.

\section{c. HISTOGRAM BASED METHOD}

Histogram based methods are very efficient when compared to other image segmentation methods because they typically require only one pass through the pixels. In this technique, a histogram is computed from all of the pixels in the image, and the peaks and valleys in the histogram are used to locate the clusters in the image.

\section{EDGE DETECTION}

The first region-growing method was the seeded region growing method. This method takes a set of seeds as input along with the image. The seeds mark each of the objects to be segmented. The regions are iteratively grown by comparing all unallocated neighbouring pixels to the regions.

\section{E.WATERSHED TRANSFORMATION}

The watershed transform has interesting properties that make it useful for much different image segmentation application. The watershed transformation is a powerful tool for image segmentation based on mathematical morphology. A watershed transformation as a means to separating overlapping objects. We can consider the image as a landscape or topographic relief where the gray level of each pixel is interpreted as its altitude in the relief. Immersing the landscape in a lake with holes pierced in local minima, catchment basins will fill up with water starting at these local minima. At points where water coming from different basins would meet, dams are built. This process ends when the water level has reached the highest peak in the landscape. As a result, the landscape is partitioned into regions or basins separated by dams, called watershed lines or simply watersheds.

\section{F. MARKER BASED WATERSHED TRANSFORMATION}

The advantages of the watershed transformation are that it is simple, instinctive knowledge, and can be parallelized. The main drawback of this method is the over-segmentation due to the presence of many local minima. To decrease the effect of severe over-segmentation, marker-controlled watershed transformations have been proposed. These are robust and flexible methods for segmenting objects with closed contours. The internal marker and external marker are initially defined. The boundaries, even if not clearly defined, are expressed as ridges between two markers and located.

In marker-controlled watershed method to segment the image the external marker is obtained manually by drawing a circle enclosing object of our interest. The internal marker is determined automatically by combining techniques including Canny edge detection, thresholding and morphological operation. Modify the segmentation function so that it only has minima at the foreground and background marker locations. Compute the watershed transform of the modified segmentation function 
Fig 1- Flowchart for marker-controlled watershed segmentation.

\section{GAPS IN LITERATURE}

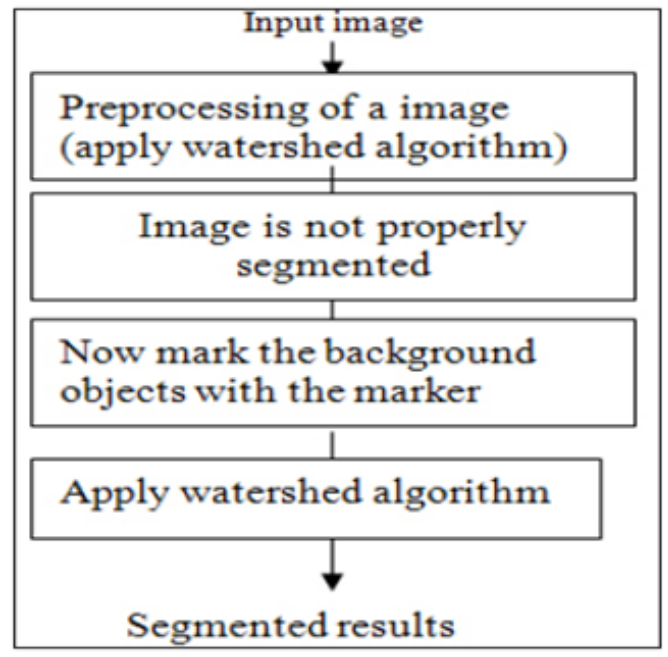

In image segmentation the objects in the image are separated and labelled for further analysis. This is usually done with the help of a wide range of image segmentation techniques. Each technique has its own advantages and disadvantages. The effectiveness of a particular image segmentation algorithm is determined with respect to a particular class of images. Generally a combination of two or more techniques is used to get the desired output for a particular application. The scale of segmentation is application specific and even the objects that are to be segmented. It is important to note that:

1. There is no universally applicable segmentation technique that is guaranteed to work on all images.

2. No segmentation technique is perfect.

The goal however is to come up with an ad-hoc technique for segmentation of high resolution aerial imagery which is a step further in image analysis. In recent years various techniques have been developed for image segmentation. One of them is watershed technique applied on images but it has various drawbacks that is over segmentation and sensitivity to noise so to overcome this we will use marker based watershed technique to overcome these problems. This technique "mark," foreground objects and background locations. The results obtained by the segmentation of the image are generally very subjective, as it depends on the information content of the image itself. The parameters to be considered for the evaluation of these results vary from image to image. Hence this makes it a very complicated process for evaluation of the results. The problem is that it's very difficult to define what a good segmentation is. It depends a lot on, amongst other things the nature of the image. Lighting conditions, noise, texture - these can all have a large impact on the results. A key problem in segmentation is that of splitting up into too few (under segmentation) or too many regions (over segmentation).

\section{Proposed algorithm}

Fig 2- Flowchart of Proposed Algorithm

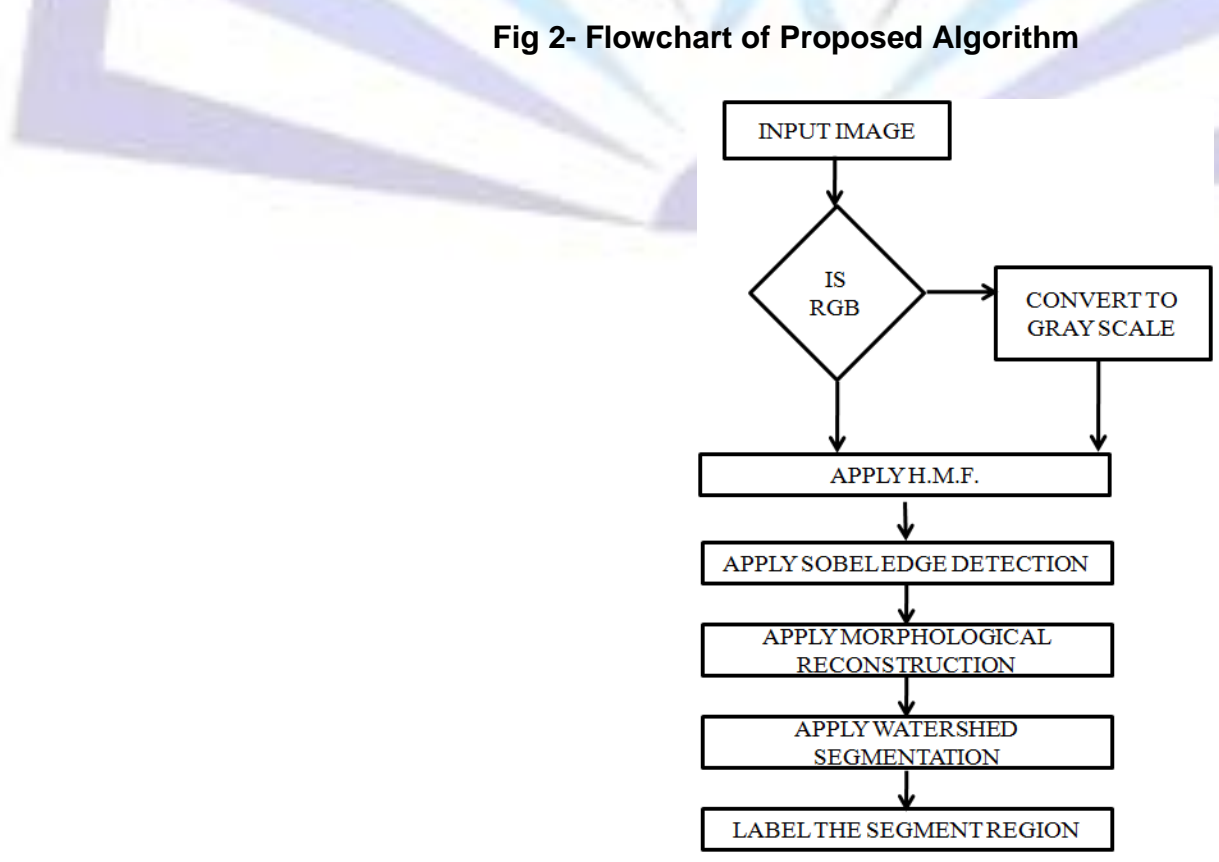


Figure 2 is showing the flowchart of the proposed algorithm. It shows the different steps which are required to implement the proposed algorithm. The main difference here is to use of the Hybrid median filter (HMF) to reduce the effect of the salt and pepper noise in efficient manner.

\section{Experimental set-up}

In order to implement the proposed algorithm; design and implementation is done in MATLAB using image processing toolbox. In order to do cross validation we have also implement the algorithm without filtering. As we know it is difficult to compare the manual seed placement algorithm with automatic seed placement objectively so only results are shown Table 1 is showing the various images which are used in this research work. Images are given along with their format and size.

Table1: format and size.

\begin{tabular}{|l|l|l|l|}
\hline Sr.No. & NAME & FORMAT & SIZE IN KB \\
\hline 1. & image1 & JPEG & 78.8 \\
\hline 2 & image2 & JPEG & 37.3 \\
\hline 3 & image3 & JPEG & 9.85 \\
\hline 4 & image4 & TIFF & 230 \\
\hline 5 & image5 & JPEG & 16.8 \\
\hline 6 & image6 & JPEG & 3.35 \\
\hline 7 & image7 & TIFF & 297 \\
\hline 8 & image8 & JPEG & 13.2 \\
\hline 9 & image9 & JPEG & 9.83 \\
\hline 10 & image10 & JPEG & 10.0 \\
\hline 11 & image11 & JPEG & 10.2 \\
\hline 12 & image12 & JPEG & 165 \\
\hline 13 & image13 & JPEG & 10.7 \\
\hline 14 & image14 & TIFF & 192 \\
\hline 15 & image15 & JPEG & 15.7 \\
\hline
\end{tabular}

\section{Experimental Results}

To justify the proposed work results are taken for the images given in table 1. Figure 3 is showing the input noisy image for justification of the proposed work.

Fig 3- Noisy image for segmentation

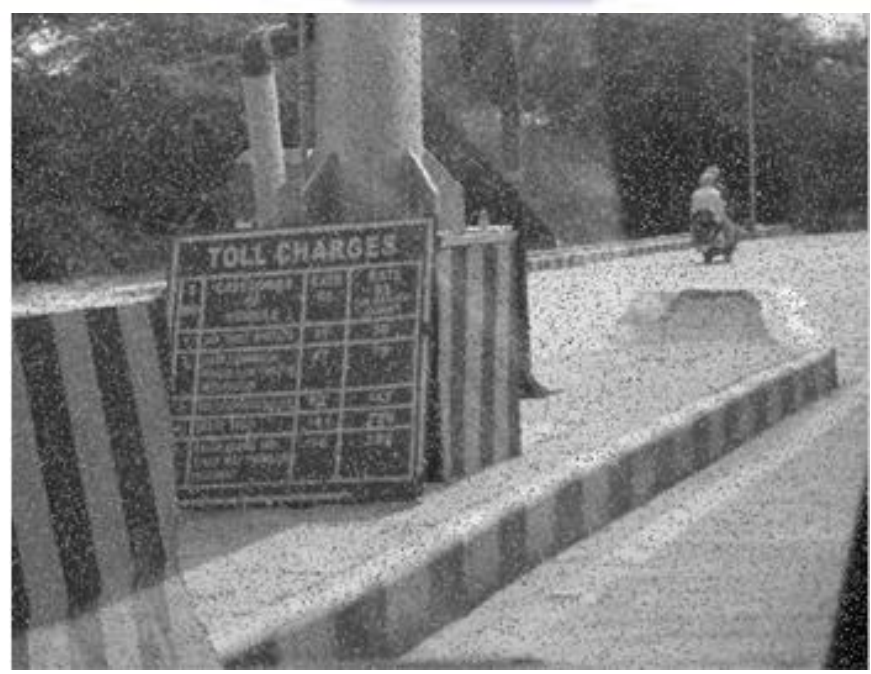


Figure 4 is showing the watershed segment image using bilateral filter based algorithm.

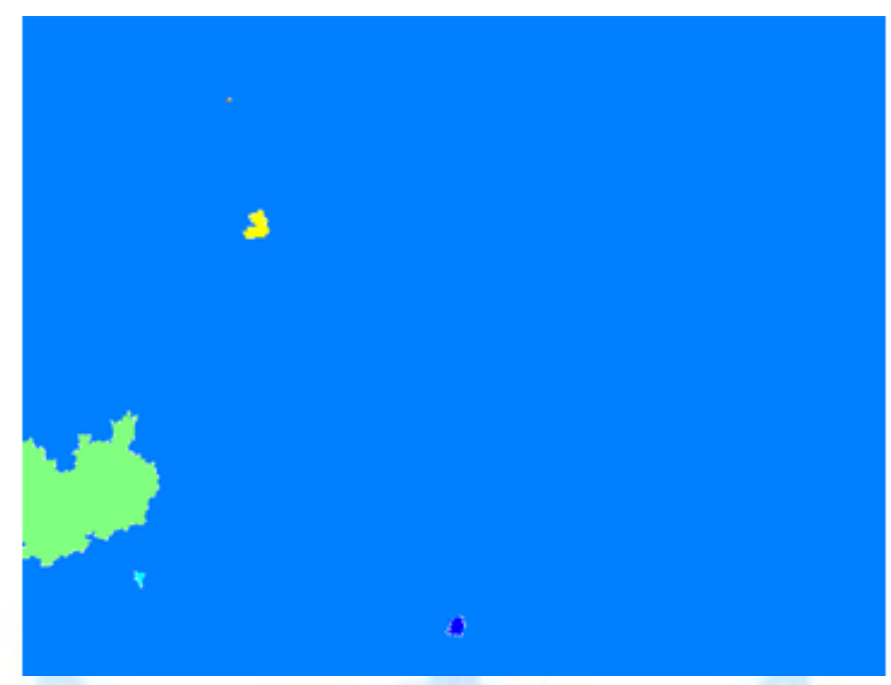

Fig 4- Watershed segmentation using bilateral filter

Figure 5 is showing the watershed segment image using hybrid median filter based algorithm.

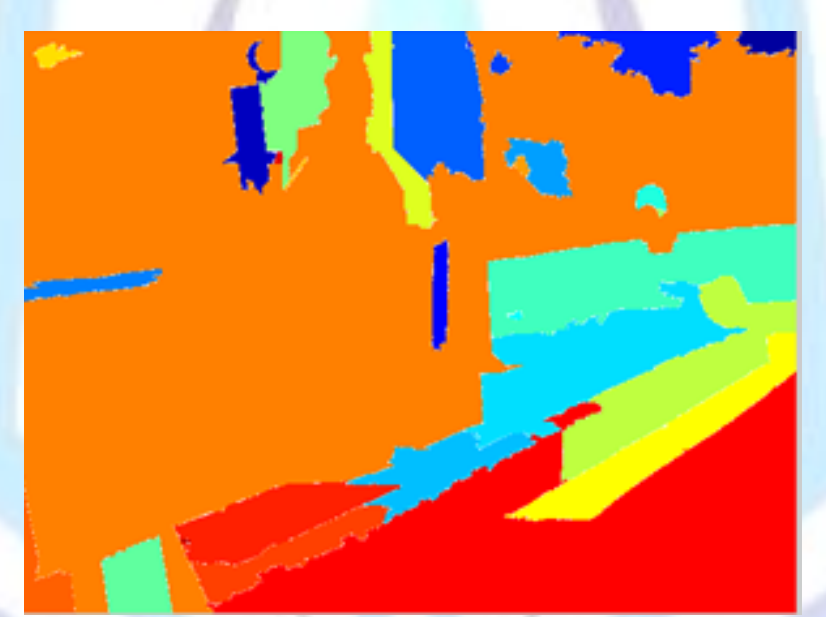

Fig 5- Watershed segmentation using hybrid median filter

Figure 6 is showing the effect of the watershed segment image on input image using bilateral filter based algorithm.

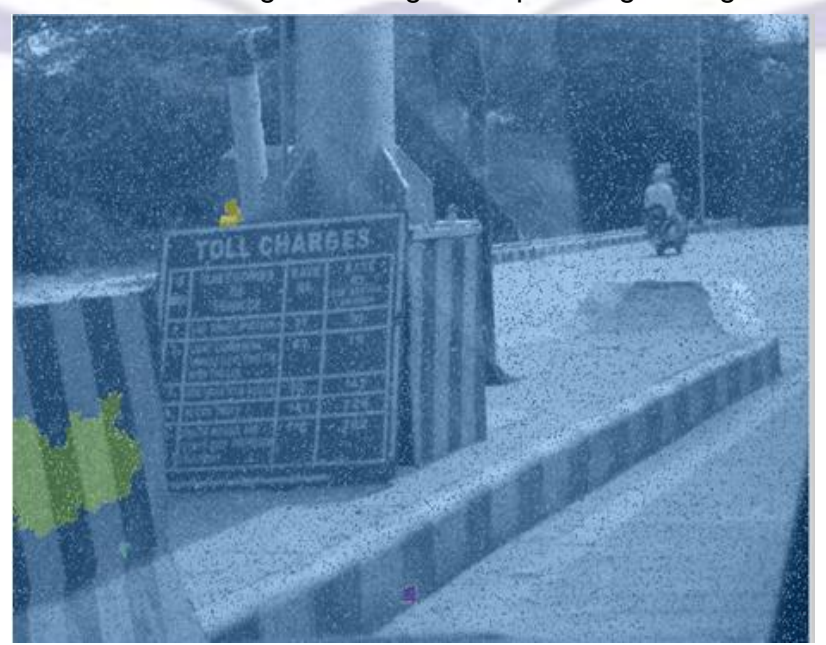

Fig 6- Watershed segmentation effect on input image using bilateral 
Figure 7 is showing the effect of the watershed segment image on input image using hybrid median filter based algorithm.

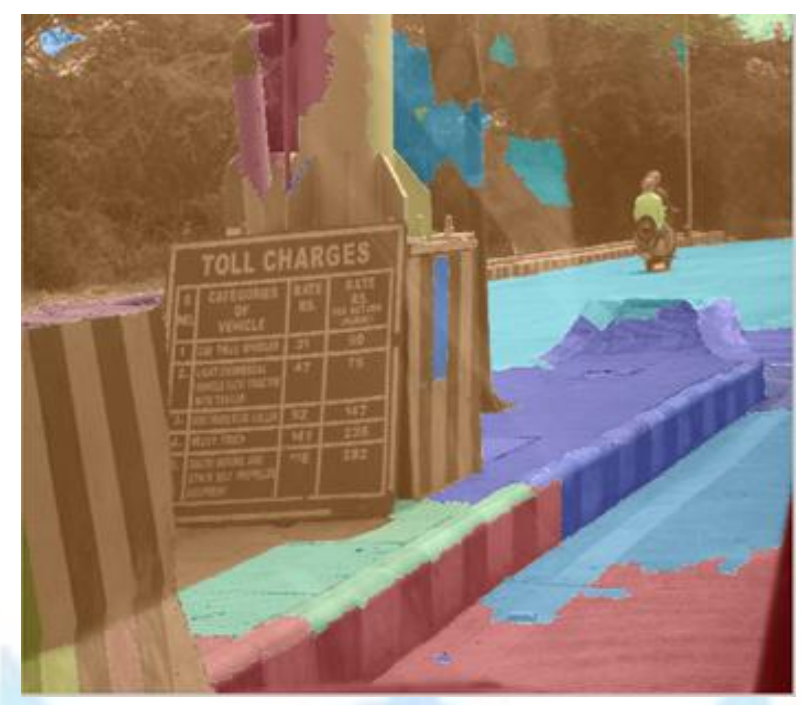

Fig 7- Watershed segmentation effect on input image using hybrid median filter

Therefore from experimental results we can see that the proposed algorithm provides quite better results than existing algorithm especially in case of noisy images.

\section{Performance evaluation}

Table 2 and Figure 8 is showing the comparative analysis of the Mean square error (MSE). As MSE need to minimize; so our goal is to reduce them MSE as much as possible. Table 2 and Figure 8 is clearly shown that MSE is less in our case therefore proposed algorithm is providing better results.

\begin{tabular}{l} 
Table 2 MSE EVALUTION TABLE \\
$\qquad$\begin{tabular}{|l|l|l|}
\hline $\begin{array}{l}\text { IMAGE } \\
\text { NAME }\end{array}$ & $\begin{array}{l}\text { WITHOUT } \\
\text { FILTER }\end{array}$ & $\begin{array}{l}\text { PROPOSED } \\
\text { ALGORITHM }\end{array}$ \\
\hline image1 & 16304 & 1895 \\
\hline image2 & 34093 & 2184 \\
\hline image3 & 32284 & 2276 \\
\hline image4 & 16391 & 1590 \\
\hline image5 & 19136 & 1764 \\
\hline image6 & 16384 & 1869 \\
\hline image7 & 21041 & 1911 \\
\hline image8 & 16020 & 1773 \\
\hline image9 & 15435 & 1803 \\
\hline image10 & 13144 & 1952 \\
\hline image11 & 18379 & 2133 \\
\hline image12 & 17501 & 1886 \\
\hline image13 & 32399 & 2045 \\
\hline image14 & 6711 & 2111 \\
\hline image15 & 16331 & 1971 \\
\hline
\end{tabular} \\
\hline
\end{tabular}




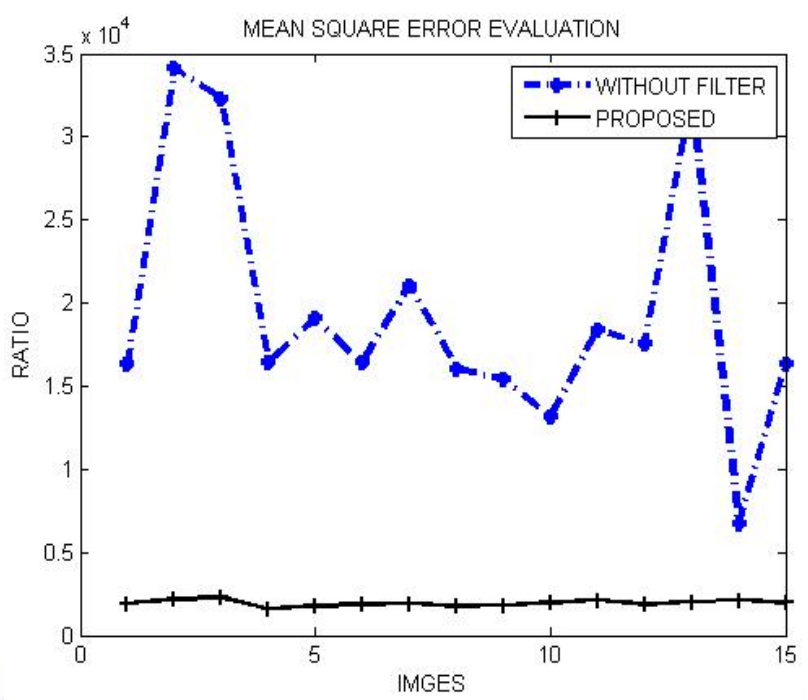

Figure 8 MEAN SQUARE ERROR (MSE) EVALUTION

Table 3 and Figure 9 is showing the comparative analysis of the Peak Signal to Noise Ratio (PSNR). As PSNR need to be maximized; so our goal is to increase PSNR as much as possible. Table 3 and Figure 9 is clearly shown that PSNR is maximum in our case therefore proposed algorithm is providing better results.

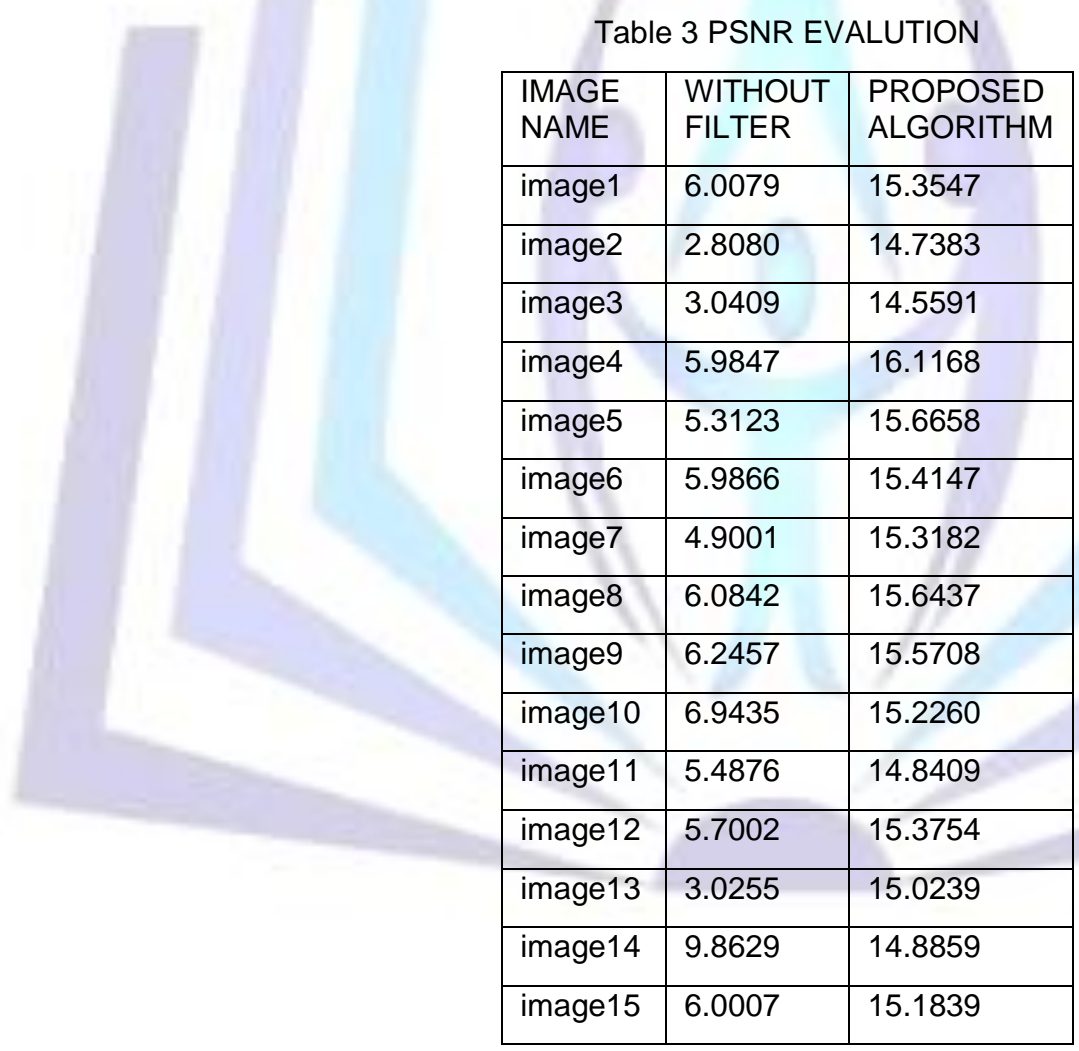




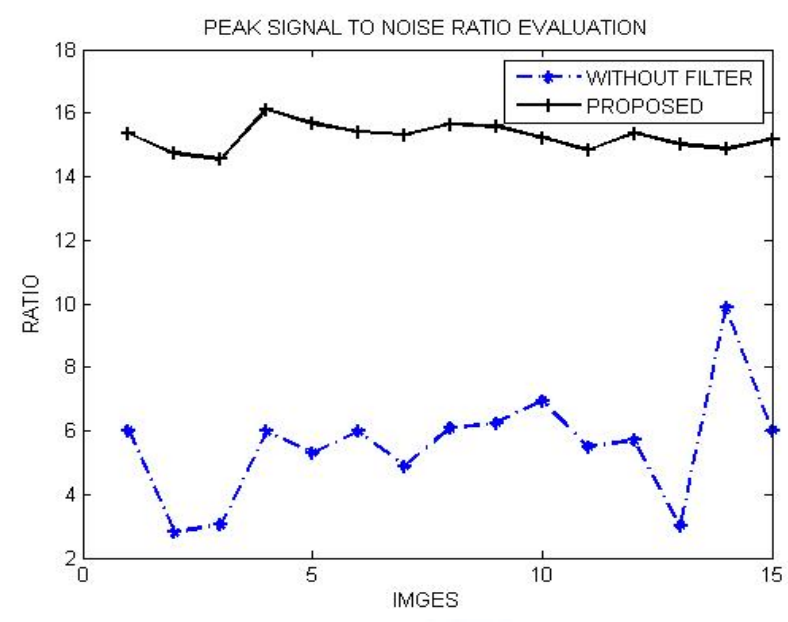

Figure 9 PSNR EVALUTION

Table 4 and Figure 10 is showing the comparative analysis of the Maximum Difference. As Maximum Difference need to be minimized; so our goal is to reduce them Maximum Difference as much as possible. Table 4 and Figure 10 are clearly shown that Maximum Difference is less in our case therefore proposed algorithm is providing better results.

Table 4 MAXIMUM DIFFERENCE

\begin{tabular}{|l|l|l|}
\hline $\begin{array}{l}\text { IMAGE } \\
\text { NAME }\end{array}$ & $\begin{array}{l}\text { WITHOUT } \\
\text { FILTER }\end{array}$ & $\begin{array}{l}\text { PROPOSED } \\
\text { ALGORITHM }\end{array}$ \\
\hline image1 & 253.9984 & 243 \\
\hline image2 & 254.9978 & 255 \\
\hline image3 & 254.9972 & 255 \\
\hline image4 & 248.9966 & 251 \\
\hline image5 & 246.9960 & 237 \\
\hline image6 & 254.9954 & 255 \\
\hline image7 & 248.9948 & 240 \\
\hline image8 & 254.9941 & 237 \\
\hline image9 & 239.9935 & 210 \\
\hline image10 & 243.9921 & 244 \\
\hline image11 & 253.9923 & 234 \\
\hline image12 & 252.9917 & 252 \\
\hline image13 & 254.9911 & 254 \\
\hline image14 & 200.9905 & 153 \\
\hline image15 & 254.9899 & 255 \\
\hline & & \\
\hline
\end{tabular}




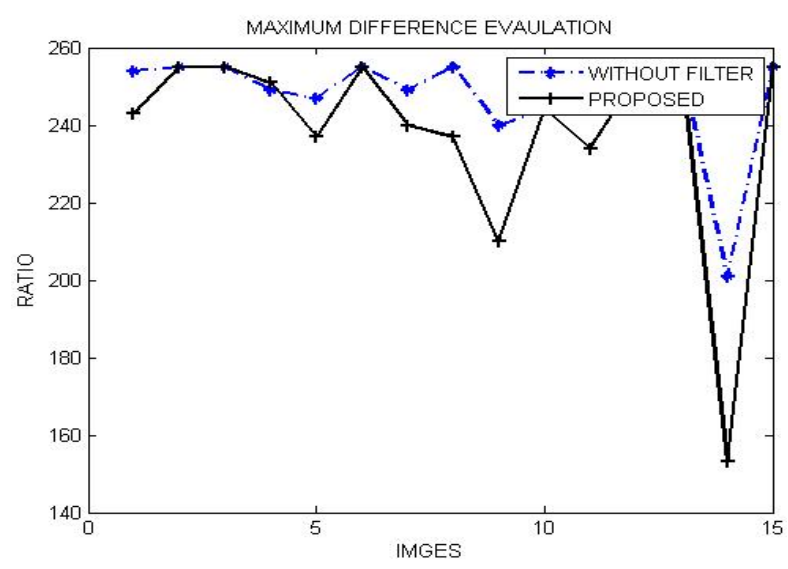

Figure 10 MAXIMUM DIFFERENCE EVALUTION

Table 5 and Figure 11 are showing the comparative analysis of the Maximum Difference. As Mean Difference needs to be minimized; so our goal is to reduce them Mean Difference as much as possible. Table 5 and Figure 11 are clearly shown that Mean Difference is less in our case therefore proposed algorithm is providing better results.

Table 5 MEAN DIFFERENCE

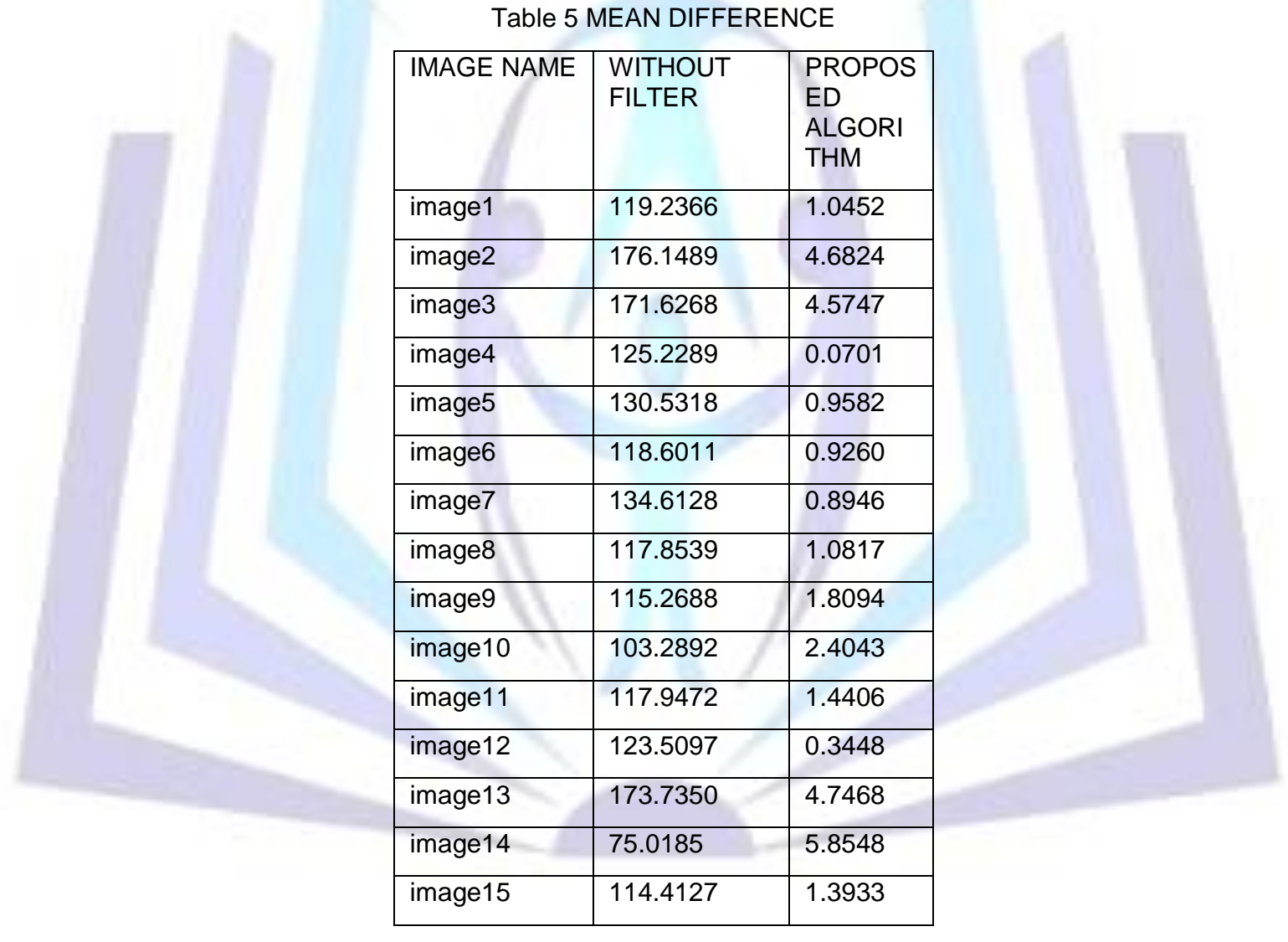




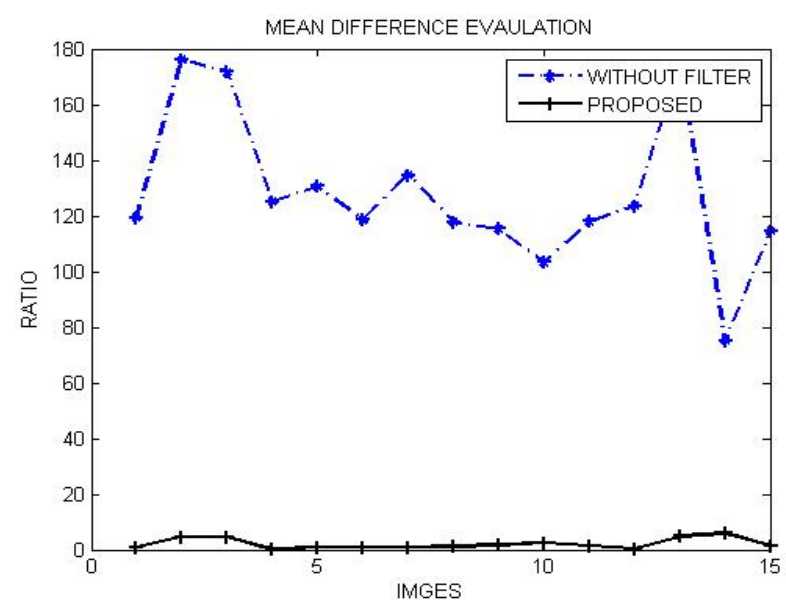

Figure 11 MEAN DIFFERENCE EVALUTION

\section{CONCLUSION}

This paper has presented a new integrated marker based watershed segmentation algorithm with hybrid medina filter. The different segmentation techniques are reviewed and found that marker based is best in most of cases because it marks the regions then segment them. However it has found that most of existing techniques uses the bilateral filter. But as it is known in prior bilateral filter is unable to reduce salt and pepper noise. So to remove this problem we will integrate marker based watershed segmentation algorithm with hybrid median filter to improve the performance of proposed algorithm. The subjective and qualitative results have shown a significant improvement over the existing algorithms. In near future we will extend this work to provide the better results even highly noise effected images i.e. having noise density around 0.7 to 0.8 .

\section{ACKNOWLEDGMENTS}

I would like to thank SSIET for funding this research. I would also like to thank Professor Ashish verma for guiding me and sharing his wealth of experience and knowledge to further my education.

Thanks to my parents who always support me.

\section{REFERENCES}

[1] Baojing ji, jianPing Lv and CaiXia Zhoa, "Improved Watershed Algorithm Based On Segmentation,",Xian institute of posts and telecommunications, pp.103-107, 2008.

[2] Boren Li and Mao Pan, "An Improved Segmentation of High Spatial Resolution Remote Sensing Image using Markerbased Watershed Algorithm," pp. 98-104, IEEE,2012.Fröhlich, B. and Plate, J. 2000. The cubic mouse: a new device for three-dimensional input. In Proceedings of the SIGCHI Conference on Human Factors in Computing Systems

[3] Chen Wei-bin and Wenzhou Zhejiang, "A New Watershed Algorithm for Cellular Image Segmentation Based on Mathematical Morphology," International Conference on Machine Vision and Human-machine Interface, vol.53, pp.2405-2414, IEEE, 2010.Sannella, M. J. 1994

[4] GuiMei Zhang and Ming-Ming Zhou, "Labelling watershed algorithm based on Morphological Reconstruction in Color Space," pp.51-55, IEEE,2011.

[5] Jun Tang, "A Color Image Segmentation algorithm Based on Region Growing,” vol.6, pp.634-637,IEEE, 2010.

[6] Li Cheng, Li Yan and Fan Yan Shangchun, "CCD infrared image segmentation using watershed algorithm," Third International Conference on Measuring Technology and Mechatronics Automation, vol.1, pp.680-683, IEEE,2011.

[7] Shiping Zhu, Xi Xia and Qingrong Zhang, "An Image Segmentation Algorithm in Image Processing Based on Threshold Segmentation," Third International IEEE Conference on Signal-Image Technologies and internet based system, IEEE, 2008.

[8] Quan Longzhe, "Automatic Segmentation method of Touching Corn Kernels in Digital Image Based on Improved Watershed Algorithm," pp.34-37, IEEE, 2011

[9] Wei Zhang, "The Marker-Based Watershed Segmentation Algorithm of Ore Image," pp.472-474, IEEE, 2011.

[10] Xiaoyan Zhang, Lichao Chen, Lihu Pan and Lizhi Xiong, "Study on the Image Segmentation Based On ICA and Watershed Algorithm," Fifth International Conference on Intelligent Computation Technology and Automation, pp. 978-912, IEEE 2012.

Yuqian Zhao, Jianxin Liu , Huifen Li and Guiyuan Li, "Watershed Algorithm for Dowels Image Segmentation," World Congress on Intelligent Control and Automation, pp7644-7648, IEEE, 2008. 